\title{
Análisis bibliométrico de la producción científica de la Universidad de Málaga en el Social Sciences Citation Index (1998-2007)
}

\author{
Alexander Maz-Machado*, Manuel Torralbo-Rodríguez*, \\ Mónica Vallejo-Ruiz**, Rafael Bracho-López*
}

Resumen: Se realiza un análisis bibliométrico de la producción de la Universidad de Málaga durante el período 1998-2007 en las revistas indexadas en el Social Sciences Citation Index, accediendo a la base de datos a través de la Web of Science. Se ha estudiado la productividad y la distribución temporal, la colaboración nacional e internacional, la producción científica por facultades y las revistas en las que se publican los artículos. Los resultados revelan un leve incremento en la productividad, así como un grado de coautoría $(2,85)$ ligeramente superior al establecido para las Ciencias Sociales en España y un buen nivel de colaboración con instituciones universitarias nacionales y extranjeras.

Palabras clave: Universidad de Málaga, indicadores bilbiométricos, Ciencias Sociales, producción científica y bibliográfica, Social Sciences Citation Index.

\section{Bibliometric analysis of scholarly production from the University of Malaga in the Social Sciences Citation Index (1998-2007)}

\begin{abstract}
A bibliometric analysis was performed of the scholarly production of the University of Malaga published during 1998-2007 in journals indexed in the Social Sciences Citation Index (as accessed through the Web of Science). This paper examines productivity and chronological distribution, national and international collaboration, scientific production by departments, and the journals in which articles were published. The results reveal a slight increase in productivity, as well as a slightly higher level of co-authorship (2.85) than the average for social sciences in Spain, along with a good level of collaboration with national and foreign academic institutions.
\end{abstract}

Keywords: University of Malaga, bibliometric indicators, Social Sciences, scholarly and bibliographic production, Social Sciences Citation Index.

* Departamento de Matemáticas, Universidad de Córdoba, España. Correo-e: ma1mamaa@uco.es, ma1torom@uco.es, rbracho@gmail.com.

** Facultad de Educación, Universidad de Murcia. Correo-e: monicavr@um.es.

Recibido: 16-10-2009; 2. ${ }^{a}$ versión: 27-1-2010; aceptado: 28-1-2010. 


\section{Introducción}

Las universidades, en su función de generar nuevos conocimientos, buscan los mecanismos más idóneos para difundirlos en la sociedad y, en particular, en la comunidad científica. Con la presente situación económica mundial, se espera que estas instituciones sean eficientes y responsables en la gestión de los recursos humanos, económicos y logísticos relacionados con la investigación. Esto crea la necesidad de conocer cuál es la producción científica universitaria en un país (Delgado y Russell, 1992), una comunidad autónoma o de una provincia, tanto de forma general como en tópicos específicos, como se refleja en la orientación de las investigaciones sobre el tema (Alonso-Arroyo y otros 2006; Campanario y otros, 1998; Maz, Torralbo y otros, 2007; Moya-Anegón y otros, 2007). Esto es importante y necesario por cuanto permite además conocer los campos científicos en los que destaca internacionalmente cada universidad.

También para las universidades es importante conocer la producción de sus investigadores en los diversos campos de la ciencia (Van Raan, 2005), aunque esto genera valoraciones que, a su vez, afectan a la evaluación de la actividad científica de los profesores de las propias universidades (Torres-Salinas y otros, 2009) y, en el caso español, repercute en los complementos salariales.

A través del análisis bibliométrico se obtienen indicadores de la actividad científica, útiles para ofrecer una visión global del trabajo realizado en los centros de investigación y puesto que las instituciones que evalúan la productividad de los investigadores, la Agencia Nacional de Evaluación de la Calidad y Acreditación (ANECA) y la Comisión Nacional de Evaluación de la Actividad Investigadora (CNEAI) señalan la publicación en las revistas indexadas en la Web of Science como criterio primordial para otorgar reconocimiento investigador, éstas se convierten en herramientas no sólo útiles sino indispensables para identificar el volumen de la producción científica de las universidades, pese a que algunos autores señalan el sesgo de estas bases de datos hacia determinados campos (Moed, 2005), en detrimento por ejemplo de las Ciencias Sociales.

La Universidad de Málaga es una institución joven con sólo 32 años de creación pero que en los últimos años viene implementando programas de fomento y desarrollo de actividades científicas, siendo el más reciente el III Plan Propio de Investigación de la Universidad de Málaga en vigor desde el año 2006. La institución cuenta con 21 centros de los cuales más de la mitad corresponden a las Ciencias Sociales, Jurídicas y Empresariales, por lo que parece pertinente analizar su presencia en la base de datos SSCI, más aún si se toma en cuenta que Moya-Anegón y otros (2005) señalan que es usual en los estudios bibliométricos de ámbito andaluz excluir los datos tanto del Social Sciences Citation Index (SSCI) como del Arts \& Humanities Citation Index (A\&HCI).

Rev. Esp. Doc. Cient., 33, 4, octubre-diciembre, 582-599, 2010. ISSN: 0210-0614. doi:10.3989/redc.2010.4.757 583 


\section{Material y métodos}

Para realizar este estudio se utilizó el JCR Social Sciences Edition y la base de datos Social Sciences Citation Index accesible a través de la Web of Science (WoS), consultándose en el mes de diciembre de 2008. El período analizado se corresponde a los años 1998 hasta 2007. El objetivo era analizar la producción en el campo de las Ciencias Sociales de los investigadores vinculados a la Universidad de Málaga (de ahora en adelante UMA) en una base de reconocido prestigio científico, cual es la SSCI.

El procedimiento para obtener las referencias consistió en analizar la información contenida en el campo Addresses y se utilizó la sentencia: "Univ. Malaga"; además en el campo Year Publisher se limitó a los años 1998-2007. Se obtuvieron 365 registros. El siguiente paso llevó a realizar un control manual para depurar aquellas referencias que no correspondieran a la UMA o al SSCI, porque pese a limitar la búsqueda a esta base de datos, en ocasiones se identificaron registros de documentos en revistas que al verificarlas únicamente corresponden a las indexadas en el JCR Science Edition, como ocurrió con Annals of Operation Research, Revista de Neurologia, Journal of Sexual Medicine, Methods and Findings in Experimental and Clinical Pharmacology, Paleobiology, Journal of Vertebrate Paleontology, European Journal of Neuroscience, Maturitas, Pain, Medical Science Research, Medical Science Monitor, Journal of Neuropsychiatry and Clinical Neurosciences, Geobios, y Dynamis entre otras. Estos registros fueron excluidos, quedando finalmente 339 referencias.

Posteriormente se realizó la búsqueda de toda la producción de las universidades andaluzas indexadas en el SSCI en el mismo período estudiado; para ello se introdujo el nombre las diez universidades en el campo Adresses, de esta forma se pudo comparar los resultados con la producción de la UMA. La información se sometió a un proceso de normalización y se calcularon los siguientes indicadores:

\section{A) Indicadores bibliométricos generales:}

- Tipo de trabajos.

- Produccción diacrónica.

- Tasa de variación interanual.

- Idioma de publicación.

- Número de producciones por facultad.

\section{B) Indicadores para la caracterización de la producción en revistas:}

- Número y porcentaje de trabajos publicados en España.

- Número y porcentaje de trabajos publicados en el extranjero.

- Número de revistas donde se publicó. 


\section{C) Indicadores para la caracterización de la colaboración:}

- Autores por artículo.

- Instituciones por artículo.

- Instituciones españolas firmantes.

- Instituciones extranjeras firmantes.

Puesto que no había uniformidad en la indicación de la filiación institucional se fijó el nivel de producción por facultades.

\section{Resultados}

\section{A) Indicadores generales}

En relación al tipo de trabajos, la producción de la UMA en los años 19982007 reflejada en el SSCI corresponde a 339 documentos, con una mayor presencia de artículos científicos (69,9\%), mientras que las cartas al editor y los biographical-item son prácticamente testimoniales (tabla I). El número de Proceedings Paper, Meeting Abstract y Book Review es muy equilibrado con pequeñas diferencias entre ellos.

\section{TABLA I}

Producción de la UMA en SSCI según el tipo de documento (1998-2007)

\begin{tabular}{l|c|c}
\hline \multicolumn{1}{c|}{ Tipo de documento } & $\mathbf{N}^{\mathbf{0}}$ de artículos & $\mathbf{\%}$ \\
\hline Article & 237 & 69,9 \\
\hline Book Review & 29 & 8,5 \\
\hline Proceedings Paper & 27 & 7,9 \\
\hline Meeting Abstract & 24 & 7,1 \\
\hline Review & 14 & 4,2 \\
\hline Editorial Material & 6 & 1,8 \\
\hline Letter & 1 & 0,3 \\
\hline Biographical-ítem & 1 & 0,3 \\
\hline Total & $\mathbf{3 3 9}$ & $\mathbf{1 0 0}$ \\
\hline
\end{tabular}

Si realizamos un análisis diacrónico de esta producción, en los 10 años analizados, se observa que si bien hay un incremento de la producción, éste es mínimo y no constante. Los datos muestran que en el lapso de una década solamente se aumentó la producción en siete documentos (figura 1). Se revela una tendencia cíclica de aumento y disminución de la producción; sin embargo, el punto mínimo de cada descenso es por lo menos igual o cercano al máximo del 
ciclo anterior. La tendencia de crecimiento con un coheficiente bajo de 0,4 y con una pendiente de 2,2 indica de forma general un incremento lento y poco continuo a lo largo de la década analizada.

FIGURA 1

Productividad de la Universidad de Málaga en el SSCI (1998-2007)

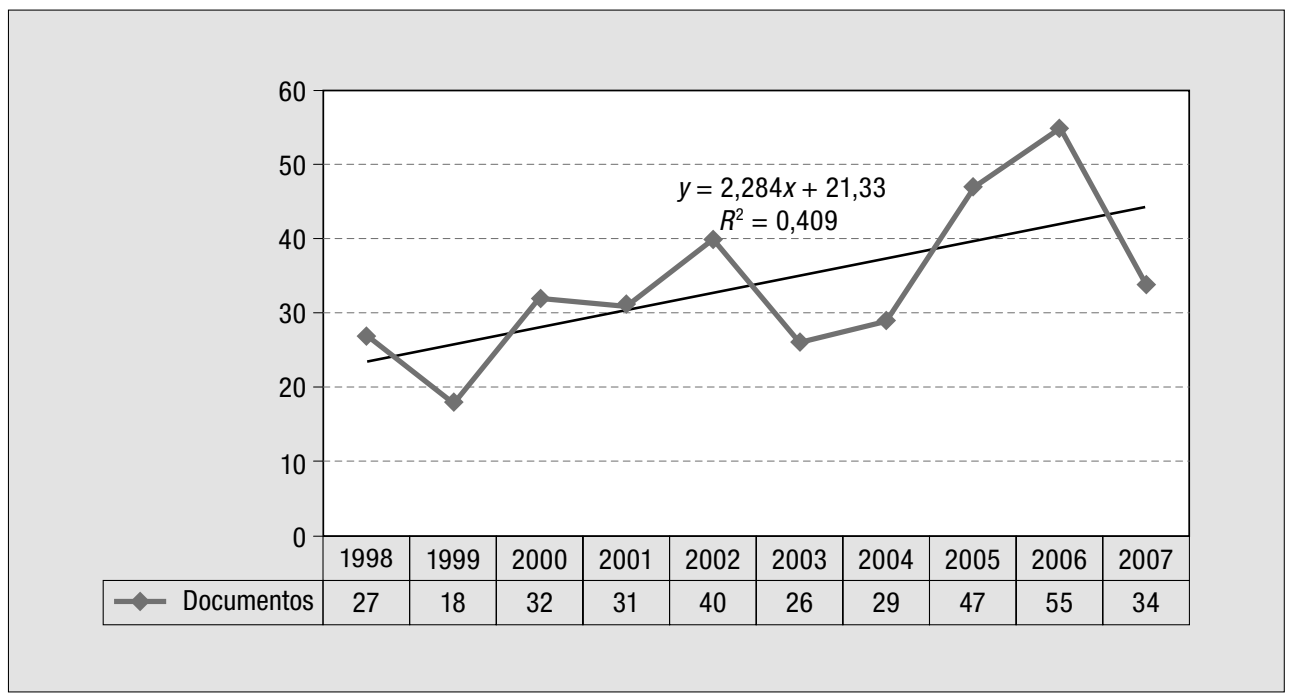

Si bien el número de trabajos es bajo, es semejante, por ejemplo, con la producción de la Universidad de Navarra, que en el período 1999-2005 tiene indizados en el WOS (SCI, SSCI y A\&HCI) 234 trabajos (Torres-Salinas y otros, 2009), mientras que la Universidad de Málaga, en la base de datos SSCI, tiene 224 trabajos. De este modo, no sería muy aventurado pensar que si a esta cifra se sumasen las producciones de las otras dos bases de datos (SCI y A\&HCI), obtendríamos una producción mayor que la de la Universidad de Navarra.

En la tabla II se destacan tres momentos relevantes en la tasa de variación interanual de la producción (TVI) en el SSCI de la UMA, en el año 2000 la TVI es máxima $(77,0)$ y en el 2007 alcanza el valor mínimo $(-38,18)$ obteniéndose una media de TVI de 9,1\%.

Al comparar esta producción de la UMA con la producción total de las universidades andaluzas en el mismo período y regristrada en el SSCI (figura 2), hallamos que éstas produjeron 1966 documentos, de las cuales 339 pertenecen a la UMA.

Se observa que en los primeros cinco años la producción de artículos de la UMA rondaba en promedio el $26,8 \%$ de la producción de las universidades andaluzas, alcanzado un máximo en el 2002. A partir de ese año disminuye el número porcentual de aportaciones respecto a Andalucía, llegando a un mínimo en el año $2007(10,9 \%)$. 


\section{TABLA II}

Tasa de variación interanual de la producción de la UMA en SSCI

\begin{tabular}{c|c|c|c}
\hline Año & $\mathbf{N}^{\mathbf{o}}$ de artículos & $\mathbf{\%}$ & TVI \\
\hline 1998 & 27 & 7,9 & - \\
\hline 1999 & 18 & 5,4 & $-33,33$ \\
\hline 2000 & 32 & 9,4 & 77,00 \\
\hline 2001 & 31 & 9,3 & $-3,12$ \\
\hline 2002 & 40 & 11,7 & 35,48 \\
\hline 2003 & 26 & 7,6 & $-36,58$ \\
\hline 2004 & 29 & 8,5 & 11,53 \\
\hline 2005 & 47 & 13,8 & 62,06 \\
\hline 2006 & 55 & 16,2 & 17,02 \\
\hline 2007 & 34 & 10,2 & $-38,18$ \\
\hline Total & $\mathbf{3 3 9}$ & $\mathbf{1 0 0 , 0 0}$ & - \\
\hline
\end{tabular}

FIGURA 2

Productividad de la UMA respecto a las universidades andaluzas en SSCI

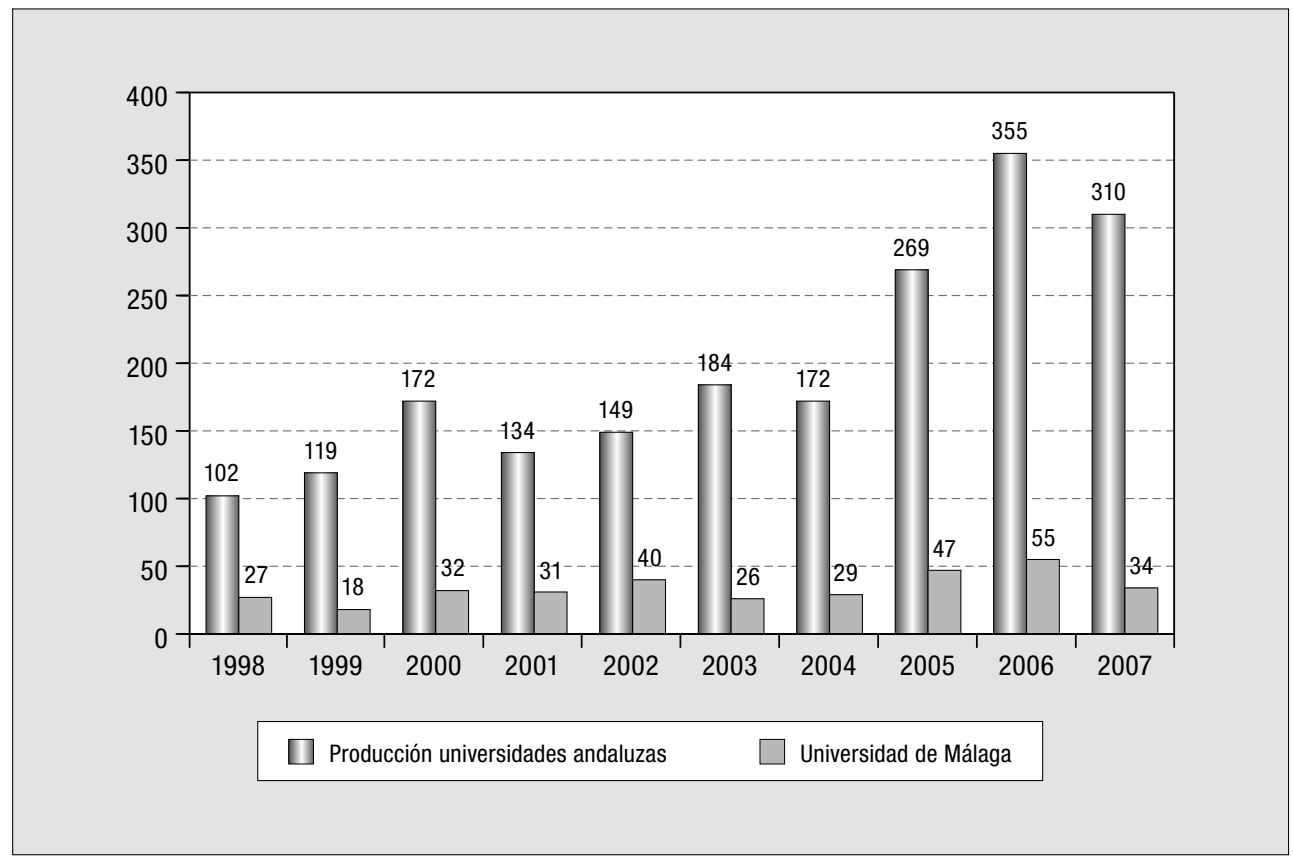


La distribución de documentos por idioma revela que el idioma de publicación es el inglés con 233 documentos (68,74\%), seguido del español con 105 $(30,97 \%)$ y un único documento en francés (0,29\%). Este dato no es muy significativo considerando el lugar de publicación de la mayoría de las revistas indexadas en esta base.

Con respecto a la filiación institucional los documentos no presentan homogeneidad, en algunos casos se indica sólo la universidad, en otros la univerisad y la facultad o el departamento, en algunos casos lo que se indica es el área de conocimiento y hasta el grupo de investigación. En 97 ocasiones únicamente se indica Univ. Málaga (28,6\%); todo esto hace que no sea posible realizar una clasificación total que sea representativa por departamentos o facultades. Sin embargo, con los datos recogidos, puede señalarse a la Facultad de Psicología como el centro más productivo con 108 documentos (31,8\%).

La producción UMA en SSCI está clasificada en 64 categorías, de ellas, el 34\% de los corresponden a Psychology Multidisciplinary, seguida a mucha distancia por Economics. En general, de las diez áreas con mayor producción 6 están relacionadas con la Psicología; todas ellas conforman el 41,85\% de las categorías, tal y como se recoge en la tabla III.

\section{TABLA III}

Distribución de la producción de la UMA en SSCI por áreas temáticas

\begin{tabular}{l|c|l|c}
\hline \multicolumn{1}{c|}{ Subject Area } & $\mathbf{N}^{\mathbf{0}}$ arts. & \multicolumn{1}{c}{ Subject Area } & $\mathbf{N}^{\mathbf{a}}$ arts. \\
\hline Psychology, Multidisciplinary & 121 & Psychology, Social & 4 \\
\hline Economics & 45 & Zoology & 3 \\
\hline Psychology, Experimental & 29 & Business & 3 \\
\hline Psychology, Clinical & 27 & Criminology \& Penology & 3 \\
\hline Environmental Studies & 24 & Education, Special & 3 \\
\hline Behavioral Sciences & 19 & Psychology, Mathematical & 3 \\
\hline Psychology & 17 & Rehabilitation & 2 \\
\hline Psychology, Biological & 17 & Communication & 2 \\
\hline $\begin{array}{l}\text { Political Science } \\
\text { Psychiatry }\end{array}$ & 16 & Computer Science, Artificial In- & telligence \\
\hline $\begin{array}{l}\text { Social Sciences, Mathematical } \\
\text { Methods }\end{array}$ & 15 & Lublic, Environmental \& Occu- & 2 \\
\hline Neurosciences & 14 & Pharmacology \& Pharmacy & 2 \\
\hline $\begin{array}{l}\text { Education \& Educational Re- } \\
\text { search }\end{array}$ & 13 & Social Issues & 2 \\
\hline Clinical Neurology & Substance Abuse & 2 \\
\hline
\end{tabular}


TABLA III (continuación)

\begin{tabular}{|c|c|c|c|}
\hline Subject Area & $\mathbf{N}^{\circ}$ arts. & Subject Area & $\mathrm{N}^{\mathrm{o}}$ arts. \\
\hline Sociology & 7 & Telecommunications & 2 \\
\hline Management & 7 & Anesthesiology & 1 \\
\hline Psychology, Educational & 7 & Anthropology & 1 \\
\hline Social Sciences, Interdisciplinary & 7 & Computer Science, Cybernetics & 1 \\
\hline $\begin{array}{l}\text { Information Science \& Library } \\
\text { Science }\end{array}$ & 7 & $\begin{array}{l}\text { Computer Science, Interdiscipli- } \\
\text { nary Applications }\end{array}$ & 1 \\
\hline Statistics \& Probability & 7 & Engineering, Industrial & 1 \\
\hline Business, Finance & 5 & Engineering, Manufacturing & 1 \\
\hline $\begin{array}{l}\text { Computer Science, Information } \\
\text { Systems }\end{array}$ & 5 & Engineering, Multidisciplinary & 1 \\
\hline Geography & 5 & Ergonomics & 1 \\
\hline Health Care Sciences \& Services & 5 & Ethics & 1 \\
\hline Health Policy \& Services & 5 & Evolutionary Biology & 1 \\
\hline $\begin{array}{l}\text { Hospitality, Leisure, Sport \& } \\
\text { Tourism }\end{array}$ & 5 & Geriatrics \& Gerontology & 1 \\
\hline Linguistics & 5 & History \& Philosophy Of Science & 1 \\
\hline $\begin{array}{l}\text { Mathematics, Interdisciplinary } \\
\text { Applications }\end{array}$ & 5 & Law & 1 \\
\hline Physiology & 5 & Medical Ethics & 1 \\
\hline Psychology, Developmental & 5 & Social Sciences, Biomedical & 1 \\
\hline $\begin{array}{l}\text { Operations Research \& Manage- } \\
\text { ment Science }\end{array}$ & 4 & Urban Studies & 1 \\
\hline Psychology, Applied & 4 & Women's Studies & 1 \\
\hline
\end{tabular}

\section{B) Indicadores para la caracterización de la producción en revistas}

La producción científica de la UMA ha sido publicada por 85 editoriales de 14 países, siendo España donde se publica el mayor número de documentos 117 (34,5\%), seguida de Inglaterra con $90(26,5)$ y Estados Unidos con 72 (21,2\%); representando entre estos tres países un total de 279 documentos (82,3\%). Los restantes 60 se reparten entre los siguientes países: Holanda (25), Alemania (15), Suiza (5), México (6), Francia (1), Colombia (2), Nueva Zelanda (2), Argentina (1), Eslovaquia (1), Irlanda (1) y Noruega (1).

Los 339 trabajos que componen la producción científica de la Universidad de Málaga en el SSCI durante el período 1998-2007 (ambos inclusive) se han publicado en un total de 166 revistas científicas, lo que hace un promedio de 2,42 documentos publicados por revista. 
La tabla IV presenta las revistas que han publicado 3 o más trabajos de la UMA, destacándose ampliamente la revista española Psicothema, editada en Oviedo y con un foco de interés en el campo de la Psicología, en la que se publica el 23\% del total, luego hay un bloque de tres revistas que juntas publican el 13,2\% de los documentos: Environmental Politics, Psicologia Conductual, y Psychological Reports. Se identificaron 12 revistas españolas en la base de datos del SSCI que habían publicado algún documento de investigadores vinculados a la Universidad de Málaga.

TABLA IV

Revistas de SSCI que ban publicado trabajos de la UMA

\begin{tabular}{|c|c|c|c|}
\hline Revista & $\mathrm{N}^{\mathrm{o}}$ arts. & Revista & $\mathbf{N}^{\circ}$ arts. \\
\hline Psicothema (España) & 78 & Aphasiology (Reino Unido) & 3 \\
\hline $\begin{array}{l}\text { Environmental Politics (Reino } \\
\text { Unido) }\end{array}$ & 16 & $\begin{array}{l}\text { Behavioral Psychology-Psicolo- } \\
\text { gia Conductual (España) }\end{array}$ & 3 \\
\hline Psicologia Conductual (España) & 15 & $\begin{array}{l}\text { Boletín de la Asociación de Geó- } \\
\text { grafos Españoles (España) }\end{array}$ & 3 \\
\hline Psychological Reports (EE.UU.) & 14 & $\begin{array}{l}\text { European Journal of Psychologi- } \\
\text { cal Assessment (EE.UU.) }\end{array}$ & 3 \\
\hline Aggressive Behavior (EE.UU.) & 9 & $\begin{array}{l}\text { International Journal of Psycho- } \\
\quad \operatorname{logy} \text { (Reino Unido) }\end{array}$ & 3 \\
\hline $\begin{array}{l}\text { Social Choice and Welfare (Ale- } \\
\text { mania) }\end{array}$ & 6 & $\begin{array}{l}\text { Investigaciones Económicas (Es- } \\
\text { paña) }\end{array}$ & 3 \\
\hline $\begin{array}{l}\text { Educational Review (Reino } \\
\text { Unido) }\end{array}$ & 5 & $\begin{array}{l}\text { Perceptual and Motor Skills } \\
\text { (EE.UU.) }\end{array}$ & 3 \\
\hline Infancia y Aprendizaje (España) & 5 & $\begin{array}{l}\text { Personality and Individual Di- } \\
\text { fferences (EE.UU.) }\end{array}$ & 3 \\
\hline Value in Health (EE.UU.) & 5 & Quality E Quantity (Países Bajos) & 3 \\
\hline Economics Letters (EE.UU.) & 4 & $\begin{array}{l}\text { Revista Mexicana de Psicología } \\
\text { (México) }\end{array}$ & 3 \\
\hline $\begin{array}{l}\text { Journal of Psychophysiology } \\
\text { (EE.UU.) }\end{array}$ & 4 & Salud Mental (España) & 3 \\
\hline $\begin{array}{l}\text { Annals of Tourism Research } \\
\text { (Reino Unido) }\end{array}$ & 3 & $\begin{array}{l}\text { Spanish Journal of Psychology } \\
\text { (España) }\end{array}$ & 3 \\
\hline
\end{tabular}

Otro de los hallazgos a destacar es la presencia de tres revistas españolas entre las 12 más utilizadas por los investigadores de esta universidad: Psicothema, Infancia y Aprendizaje y, Psicología Conductual; seguidas de un grupo heterogéneo de revistas, cuáles son: Boletín de la Asociación de Geógrafos Españoles, Investigaciones Económicas y Spanish Journal of Psychology. 
Durante el período estudiado, la producción de la UMA se ha publicado mayoritariamente en el extranjero $(65,49 \%)$, aunque la difusión por medios españoles es significativa, alcanzando un tercio del volumen total $(34,51 \%)$.

A lo largo de la década analizada, los investigadores de la UMA están variando los hábitos de publicación, puesto que se ha pasado de publicar mayoritariamente en revistas nacionales a hacerlo en un mayor porcentaje en revistas internacionales (figura 3). El número mínimo de trabajos en éstas últimas se dió en el año 1998 con 4, llegando a un máximo de 37 en el 2005, si bien ha tenido un ligero descenso en el 2007. En el caso de las revistas nacionales, el proceso ha sido contrario se partió con el máximo número de trabajos en 1998 con 23, y se llegó a valores mínimos en el año 2003 con sólo 3. A partir del año 1999 el número de trabajos publicados en revistas internacionales es mayor que el de las revistas nacionales.

FIGURA 3

Trabajos publicados en revistas españolas y del resto del mundo

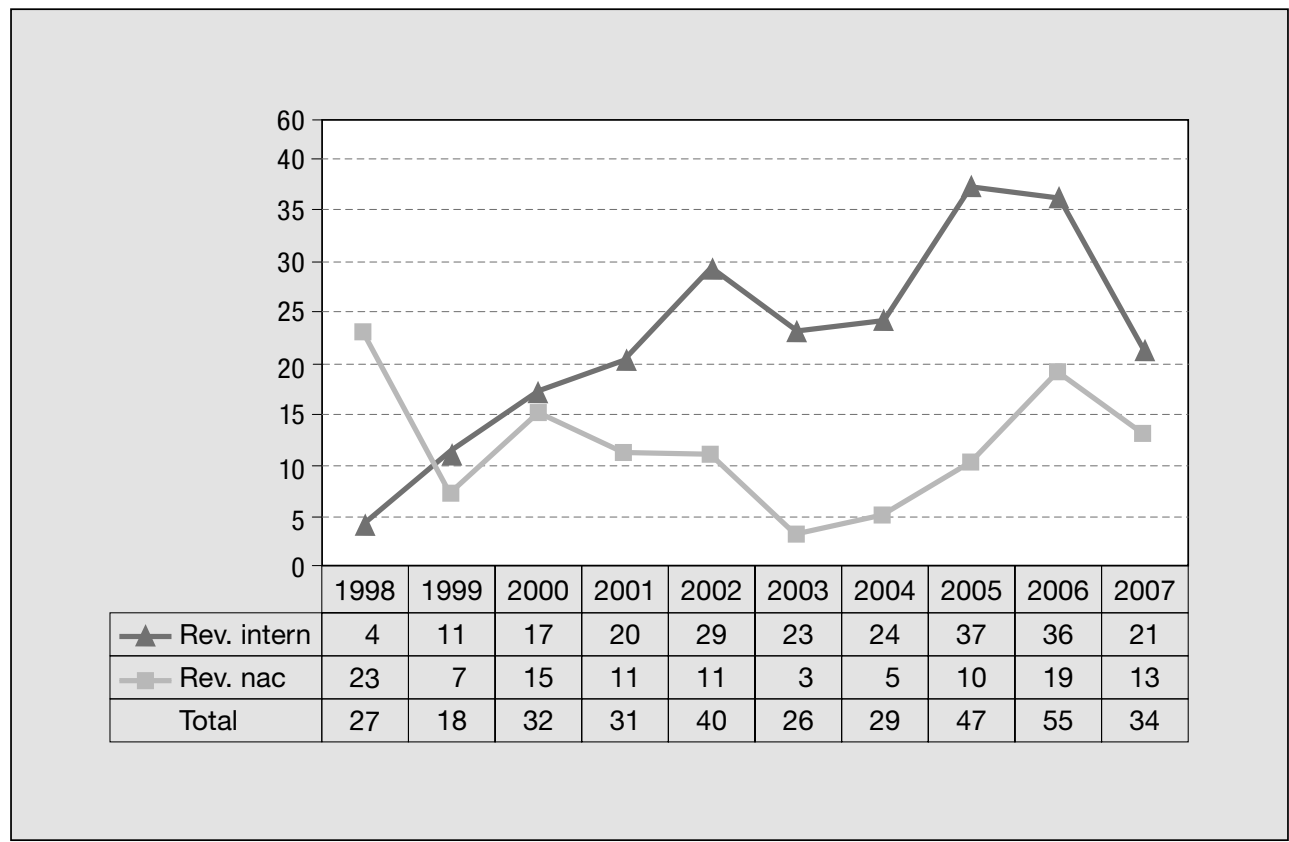

En el año 2007, la UMA publica 34 documentos en 25 revistas. La tabla V presenta el factor de impacto de estas revistas y revela que sólo seis revistas publicaron más de un documento. El 64\% de las revistas en las que se publicaron tienen un factor de impacto menor que 1. 
TABLA V

Factor de impacto en 2007 de las revistas donde publica la UMA

\begin{tabular}{|c|c|c|}
\hline Revista & FI 2007 & $\begin{array}{c}\mathrm{N}^{\mathrm{o}} \text { de artículos } \\
\text { publicados }\end{array}$ \\
\hline Behaviour Research and Therapy & 3,600 & 1 \\
\hline Annals of Behavioral Medicine & 2,929 & 1 \\
\hline Crime \& Delinquency & 1,796 & 1 \\
\hline Addictive Behaviors & 1,752 & 1 \\
\hline Cognition E Emotion & 1,511 & 1 \\
\hline Personality and Individual Differences & 1,400 & 1 \\
\hline Computers in Human Behavior & 1,344 & 1 \\
\hline Quarterly Journal of Experimental Psychology & 1,104 & 1 \\
\hline Psicothema & 0,965 & 4 \\
\hline Regional Science and Urban Economics & 0,885 & 1 \\
\hline Environmental Values & 0,741 & 1 \\
\hline Online Information Review & 0,671 & 1 \\
\hline European Psychiatry & 0,608 & 1 \\
\hline Spanish Journal of Psychology & 0,589 & 2 \\
\hline Social Choice and Welfare & 0,493 & 1 \\
\hline International Journal of Information Management & 0,451 & 1 \\
\hline Human Factors and Ergonomics in Manufacturing & 0,420 & 1 \\
\hline Psychological Reports & 0,353 & 1 \\
\hline Empirical Economics & 0,280 & 2 \\
\hline Investigaciones Económicas & 0,268 & 3 \\
\hline Quality \& Quantity & 0,225 & 2 \\
\hline Boletin de la Asociacion de Geografos Espanoles & 0,072 & 1 \\
\hline Environmental Politics & 0,756 & 1 \\
\hline Infancia y Aprendizaje & $0^{*}$ & 2 \\
\hline Spanish Economic Review & $0^{*}$ & 1 \\
\hline
\end{tabular}

* Estas revistas aún no tenían factor de impacto para el año 2007.

Un aspecto primordial de toda producción científica es el impacto que ésta tiene en la comunidad internacional y si bien la producción de la UMA en el SSCI no es muy alta, si ha tenido repercusión a nivel de citaciones. En la tabla VI se presenta el listado de los autores de trabajos que han recibido 15 o más citaciones en el WOS. Se observa que de los 18 documentos más citados, ocho corresponden a algunos de los autores más productivos reseñados en la tabla VIII. 
TABLA VI

Listado de autores de trabajos más citados

\begin{tabular}{|c|c|c|c|}
\hline Autores & Revista & Año & $\begin{array}{c}\mathbf{N}^{\mathrm{o}} \text { de } \\
\text { citaciones }\end{array}$ \\
\hline $\begin{array}{l}\text { Kulisevsky, J.; García C.; Berthier, } \\
\text { Ml., et al. }\end{array}$ & Movement Disorders & 2000 & 55 \\
\hline Hidalgo, M. C.; Hernández, B. & J Environ Psychol & 2001 & 43 \\
\hline $\begin{array}{l}\text { López, F. J.; Shanks, Dr.; Almaraz, J., } \\
\text { et al. }\end{array}$ & J Exp Psychol-Learn Mem Cogn & 1998 & 35 \\
\hline Dietsch, M.; Lozano-Vivas, A. & J Banking \& Finance & 2000 & 32 \\
\hline $\begin{array}{l}\text { Berthier, M. L.; Kulisevsky, J.; Giro- } \\
\text { nell, A., et al. }\end{array}$ & Neuropsych Neuropsych Behav & 2001 & 31 \\
\hline Maldonado, E.; Navarro, J. F. & Pharmacological Research & 2001 & 28 \\
\hline $\begin{array}{l}\text { Capra, Cm.; Goeree, J. K.; Gómez, } \\
\text { R. et al. }\end{array}$ & American Economic Review & 1999 & 25 \\
\hline $\begin{array}{l}\text { Lozano-Vivas, A.; Pastor, J. T.; Pas- } \\
\text { tor, J. M. }\end{array}$ & J Productivity Analysis & 2002 & 22 \\
\hline $\begin{array}{l}\text { Rohleder, N.; Wolf, J. M.; Maldona- } \\
\text { do, E. F., et al. }\end{array}$ & Psychophysiology & 2006 & 21 \\
\hline Salguero, R. A.; Moran, R. & Addiction & 2002 & 20 \\
\hline $\begin{array}{l}\text { Ramírez-Maestre, C.; Martínez, A.; } \\
\text { Zarazaga, R. E. }\end{array}$ & J Behavioral Medicine & 2004 & 19 \\
\hline $\begin{array}{l}\text { García-Pando, A.; Del Pozo, J.; Sán- } \\
\text { chez, A.; et al. }\end{array}$ & J Clinical Psychiatry & 2002 & 19 \\
\hline $\begin{array}{l}\text { Fernández-Berrocal, P.; Extremera, } \\
\text { N.; Ramos, N. }\end{array}$ & Psychological Reports & 2004 & 18 \\
\hline $\begin{array}{l}\text { Extremera, N.; Fernández-Berro- } \\
\text { cal, P. }\end{array}$ & Psychological Reports & 2002 & 18 \\
\hline $\begin{array}{l}\text { Kumbhakar, Sc.; Lozano, A.; Lovell, } \\
\text { Cak, et al. }\end{array}$ & J Money Credit Bank & 2001 & 18 \\
\hline Cobos, Pl.; López, Fj.; Cano, A., et al. & J Exp Psychol-Anim Beh Process & 2002 & 17 \\
\hline Berthier, Ml. & Drugs \& Aging & 2005 & 16 \\
\hline $\begin{array}{l}\text { Extremera, N.; Fernández-Berro- } \\
\text { cal, P. }\end{array}$ & Pers Individ Differ & 2005 & 15 \\
\hline
\end{tabular}

Los datos evidencian que los trabajos más citados corresponden a aquellos publicados en revistas internacionales en inglés y, mayoritariamente, después del año 2000; por otra parte, es destacable que un artículo publicado en el año 2006 ha recibido 21 citaciones en sólo dos años mostrando una cierta inmediatez en la citación. 


\section{C) Indicadores para la caracterización de la colaboración:}

Para los indicadores de colaboración se tomaron en cuenta la producción de todos los tipos de documentos. Hay un total de 967 firmas para los 339 documentos publicados. La tabla VII señala que 2 ó 3 investigadores firman más de la mitad $(54,88 \%)$ de todos los documentos de la UMA en el SSCI.

\section{TABLA VII}

Autores firmantes por trabajo

\begin{tabular}{c|c|c|c}
\hline $\mathbf{N}^{\mathbf{0}}$ de autores & Autores & Porcentaje (\%) & Total de firmas \\
\hline 1 & 65 & 19,18 & 65 \\
\hline 2 & 101 & 29,79 & 202 \\
\hline 3 & 85 & 25,09 & 255 \\
\hline 4 & 34 & 10,04 & 136 \\
\hline 5 & 30 & 8,85 & 150 \\
\hline 6 & 16 & 4,71 & 96 \\
\hline 7 & 3 & 0,88 & 21 \\
\hline 8 & 3 & 0,88 & 24 \\
\hline 9 & 2 & 0,58 & 18 \\
\hline Total & $\mathbf{3 3 9}$ & $\mathbf{1 0 0}$ & $\mathbf{9 6 7}$ \\
\hline
\end{tabular}

El índice de colaboración viene dado por la formula $I C=n^{\circ}$ firmas $/ n^{\circ}$ de artículos, en este caso el IC de los autores en estos artículos es de 2,85. Este valor es ligeramente mayor que los índices que Bordons y Gómez (1997) establecen para las Ciencias Sociales en España (2 firmas por trabajo).

La producción de la UMA la firman 339 autores, de ellos los más productivos y pertenencientes a la propia universidad se presentan en la tabla VIII. Destaca José Francisco Navarro como el autor de mayor productividad con el 12,09\% del total. Este autor dobla la producción del segundo, Pablo Fernández-Berrocal. Se observa que cinco autores producen el 30,94\% de todos los documentos de la UMA en el SSCI. Considerando únicamente la primera firma de los trabajos, los cinco autores más productivos serían los mismos pero con diferentes valores: Navarro (19), Fernández-Berrocal (7), Blanca (7), Extremera (6) y Maldonado (5).

Destaca la permanente colaboración entre algunos de los autores, por ejemplo Dávila, Martín-López y Pedraza han firmado toda su producción con Navarro, mientras que Fernández-Berrocal lo ha hecho con Extremera en 14 ocasiones.

Otro hallazgo es que los investigadores de la UMA firman institucionalmente de forma individual el $52,5 \%$ de su producción, mientras que en un 33,3\% lo hace con otra única institución y el 10,32\% es con otras dos. El restante 3,83\% es firmado con tres o más. Entre las instituciones firmantes se tienen universida- 


\section{TABLA VIII}

Autores más productivos de la UMA en SSCI (1998-2007)

\begin{tabular}{|c|c|c|c|}
\hline Autor & Centro/Departamento & $\mathrm{N}^{\circ}$ arts. & $\%$ \\
\hline Navarro, José Francisco & Dept. Psicol. Básica Psicobiol \& Metodol & 41 & 12,09 \\
\hline Fernández-Berrocal, Pablo & Dept. Psicol. Básica Psicobiol \& Metodol & 19 & 5,60 \\
\hline Extremera, Natalio & $\begin{array}{l}\text { Psicol. Soci., Antr. Social, Trab Soc \& Serv } \\
\text { Soc }\end{array}$ & 17 & 5,01 \\
\hline Blanca, M. a José & Dept. Psicol. Básica Psicobiol \& Metodol & 16 & 4,71 \\
\hline Maldonado, Enrique & Dept. Psicol. Básica Psicobiol \& Metodol & 12 & 3,53 \\
\hline Dávila, Guadalupe & Dept. Psicol. Básica Psicobiol \& Metodol & 9 & 2,65 \\
\hline Pedraza, Carmen & Fac. Psicología & 9 & 2,65 \\
\hline Valero-Aguayo, Luis & Fac. Psicología & 8 & 2,35 \\
\hline Arias-Maldonado, Manuel & Dept. Ciencia Polít. \& Derecho Int Públ & 8 & 2,35 \\
\hline Berrocal, C. & Dept. Pers. Eval \& Trat. Psciol & 8 & 2,35 \\
\hline Hernández-Mendo, Antonio & $\begin{array}{l}\text { Psicol. Soci., Antr. Social, Trab Soc \& Serv } \\
\text { Soc }\end{array}$ & 8 & 2,35 \\
\hline Lozano-Vivas, Ana & Dept. Teoría e Historia Económica & 7 & 2,06 \\
\hline Almaraz, Julián & Dept. Psicol. Básica Psicobiol \& Metodol & 6 & 1,76 \\
\hline López, Francisco José & Dept. Psicol. Básica Psicobiol \& Metodol & 6 & 1,76 \\
\hline Martín-López, Mercedes & Dept. Psicol. Básica Psicobiol \& Metodol & 6 & 1,76 \\
\hline Pelegrina, Manuel & Dept. Psicol. Básica Psicobiol \& Metodol & 6 & 1,76 \\
\hline Rubio, Sandra & Dept. Psicol. Básica Psicobiol \& Metodol & 6 & 1,76 \\
\hline Valencia, Ángel & Dept. Ciencia Polít. \& Derecho Int Públ & 6 & 1,76 \\
\hline Trianes, M. ${ }^{\text {a }}$ Victoria & Dept. de Psicol. Evol. y de la Educación & 6 & 1,76 \\
\hline Vera, Francisca M. $^{\mathrm{a}}$ & Dept. Psicol. Básica Psicobiol \& Metodol & 6 & 1,76 \\
\hline
\end{tabular}

des, centros hospitalarios, laboratorios farmacéuticos, museos y dependencias de diversas comunidades autónomas.

La colaboración internacional de la actividad investigadora de la UMA se refleja en que 67 documentos de su producción son firmados con instituciones universitarias extranjeras. Si bien el número de colaboraciones no es muy alto, supone un 19,76\% del total de documentos, éstas se realizan especialmente con universidades europeas y norteamericanas. El mayor número de colaboraciones es con la University College London, la Universidad de Pisa, la Universidad de Virginia y la Universidad Washington \& Lee. En la tabla IX se presentan las universidades extranjeras que han publicado dos o más documentos con la UMA.

En la producción de artículos de la UMA, estos tienen coautoría con investigadores de 49 instituciones universitarias extranjeras de 13 países. La colaboración con instituciones de norteamérica es máxima con el 38,29\% del total. 
TABLA IX

Universidades extranjeras que publican con la UMA en el SSCI (1998-2007)

\begin{tabular}{l|c}
\hline \multicolumn{1}{c|}{ Institución } & $\mathbf{N}^{\mathbf{0}}$ de artículos \\
\hline University College London & 3 \\
\hline Universidad de Pisa & 3 \\
\hline Universidad de Virginia & 3 \\
\hline Universidad Washington \& Lee & 3 \\
\hline Universidad Católica de Lovaina & 2 \\
\hline Universidad de Florida & 2 \\
\hline Universidad de Amsterdam & 2 \\
\hline Universidad de California-Los Ángeles & 2 \\
\hline Universidad de Londres-London School Economic \& Politic Science & 2 \\
\hline Universidad de Texas & 2 \\
\hline Universidad de Utrecht & 2 \\
\hline Universidad de Yale & 2 \\
\hline Universidad de Nottingham & 2 \\
\hline
\end{tabular}

En cuanto a la coautoría con otras universidades españolas, se obtuvo que 104 documentos son firmados por la UMA y por lo menos una universidad española. En algunas ocasiones la autoría corresponde a la vez a la UMA junto a universidades nacionales y extranjeras. Esta colaboración solamente existe con 30 universidades españolas (tabla X).

\section{TABLA X}

Universidades españolas que publican con la UMA en SSCI (1998-2007)

\begin{tabular}{l|c|l|c}
\hline \multicolumn{1}{c|}{ Universidad Firmante } & $\mathbf{N}^{\mathbf{0}}$ arts. & \multicolumn{1}{c}{ Universidad Firmante } & $\mathbf{N}^{\mathbf{a r t s}}$ \\
\hline Universidad de Granada & 15 & Universidad de Salamanca & 3 \\
\hline Universidad de Barcelona & 14 & Universidad de la Coruna & 2 \\
\hline Universidad de Oviedo & 9 & Universidad Miguel Hernández & 2 \\
\hline Universidad de Valencia & 8 & Universidad Politécnica de Cataluña & 2 \\
\hline $\begin{array}{l}\text { Universidad de Complutense Ma- } \\
\text { drid }\end{array}$ & 8 & $\begin{array}{c}\text { Universidad de Santiago de Com- } \\
\text { postela }\end{array}$ & 2 \\
\hline $\begin{array}{l}\text { Universidad del País Vasco } \\
\text { Universidad de La Laguna }\end{array}$ & 5 & Universidad de Alicante & 1 \\
\hline $\begin{array}{c}\text { Universidad Nacional de Educación } \\
\text { a Distancia (UNED) }\end{array}$ & 5 & Universidad de Girona & 1 \\
\hline
\end{tabular}


TABLA X (continuación)

\begin{tabular}{l|c|l|c}
\hline \multicolumn{1}{c|}{ Universidad Firmante } & $\mathbf{N}^{\mathbf{0}}$ arts. & \multicolumn{1}{c}{ Universidad Firmante } & $\mathbf{N}^{\mathbf{a}}$ arts. \\
\hline Universidad de Almería & 4 & Universidad de Lleida & 1 \\
\hline Universidad Carlos III Madrid & 4 & Universidad de Navarra & 1 \\
\hline Universidad Autónoma Barcelona & 4 & Universidad Ramón Llull & 1 \\
\hline Universidad de Sevilla & 4 & Universidad Rovira i Virgili & 1 \\
\hline Universidad Autónoma Madrid & 3 & Universidad San Pablo CEU & 1 \\
\hline Universidad de Jaén & 3 & Universidad de Valladolid & 1 \\
\hline Universidad de Las Palmas & 3 & Universidad de Zaragoza & 1 \\
\hline
\end{tabular}

El mayor grado de colaboración se da con la Universidad de Granada seguida de las de Barcelona, Oviedo y Valencia. La coautoría con las universidades andaluzas es de 27 documentos (22,8\%), mientras que el total de la coautoría nacional es de 118.

La figura 4 representa la red de colaboración universitaria española en la coautoría de documentos de la UMA en el SSCI desde el año 1998 hasta el 2007 inclusive, el tamaño del nodo representa el número de documentos.

\section{FIGURA 4}

Red española de colaboración universitaria de la UMA en SSCI (1998-2007)

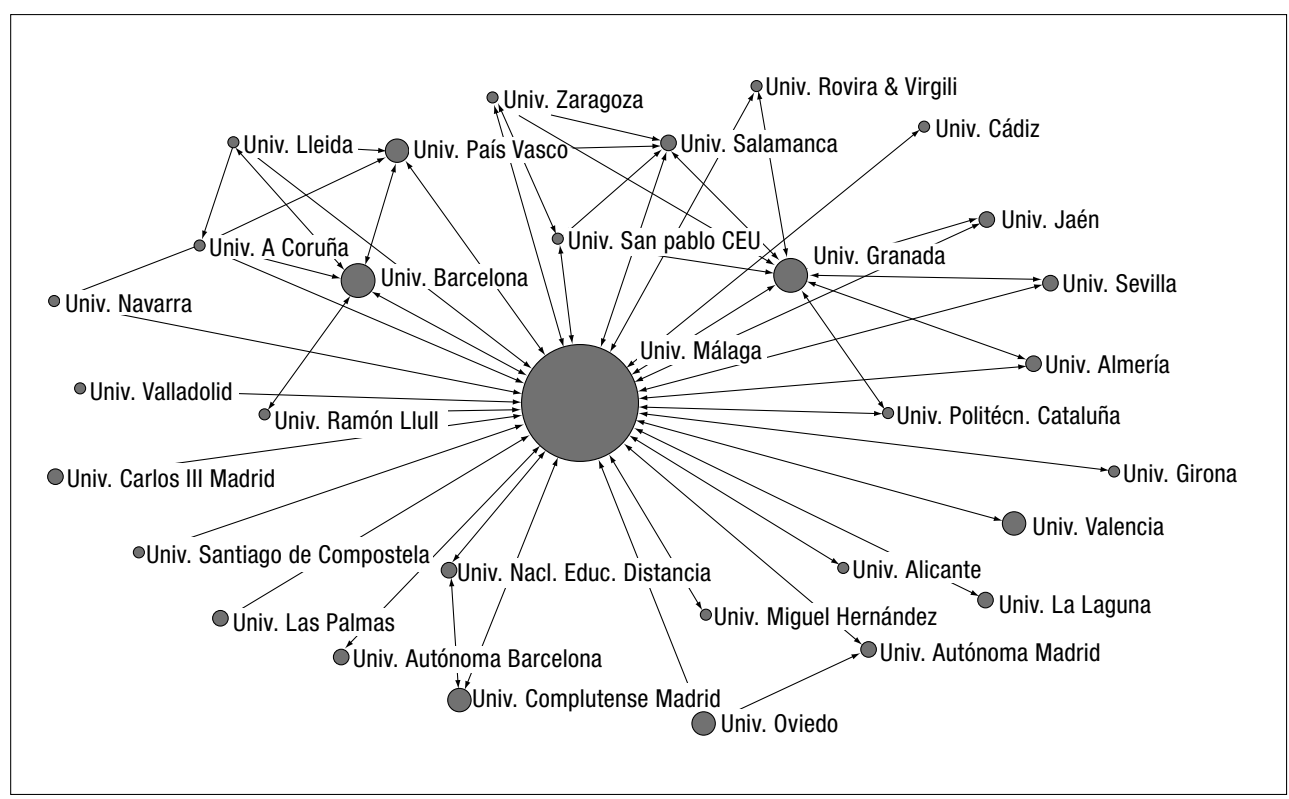


Esta red de colaboración indica que el valor de intermediación de la Universidad de Málaga es de 791,833 le siguen las universidades de Granada con 25,000, del País Vasco, de Barcelona y de Salamanca, estas últimas con 3,333.

\section{Conclusiones}

Se ha presentado una descripción de la producción de la UMA indexada en el SSCI en los últimos diez años, hallándose que ésta ha sufrido altibajos en el período 1998-2007, presentando los mayores volúmenes de producción en el lapso 2005-2007, aunque si bien ha llegado a duplicar la producción del año 1998, ha visto disminuido el volumen respecto al total de la producción de las universidades andaluzas llegando a valores mínimos en los últimos años.

Los artículos copan la producción de la UMA a lo largo del período analizado, presentando en los últimos años un incremento en la publicación de proceeding paper y meeting abstract. Sin embargo, esta producción es baja en comparación con otras universidades españolas.

Se constató que no hay uniformidad en la descripción de la filiación institucional en cuanto a los centros o departamentos de los autores; así como en la forma de firmar los documentos, presentando un mismo autor variaciones en el tiempo lo que dificulta su estudio bibliométrico y puede llegar a generar distorsiones en algunos resultados. Pese a esto, la Facultad de Psicología emerge como la gran productora de la UMA en el SSCI y José Francisco Navarro como el autor más prolífico bajo los referentes estudiados.

Los investigadores de la UMA siguen la tendencia nacional a publicar en revistas internacionales indexadas en el SSCI $(65,49 \%)$, si bien siguen publicando en revistas nacionales aunque en menor medida (34,51\%). Entre las revistas, destaca la española Psicothema como la mayor difusora de la investigación de la UMA en SSCI.

Es importante el grado de colaboración tanto a nivel institucional con centros españoles y extranjeros como a nivel de coautoría, siendo esta última mayor que la esperada para las Ciencias Sociales en España. Finalmente se aprecia un especial acercamiento hacia la colaboración con las universidades norteamericanas $\mathrm{y}$, es casi inexistente, con las universidades latinoamericanas.

\section{Bibliografía}

Alonso-Arroyo, A.; Pulgarín, A., y Gil-Leiva, I. (2006). Análisis bibliométrico de la producción científica de la Universidad Politécnica de Valencia 1973-2001. Revista Española de Documentación Científica, 29 (3), 345-363.

Bordons, M., y Gómez, I. (1997). La actividad científica española a través de indicadores bibliométricos en el período 1990-93. Revista General de Información y Documentación, 7 (2), 69-86. 
Campanario, J. M.; Cabos, W., y Hidalgo, M. A. (1998). El impacto de la producción científica de la Universidad de Alcalá de Henares. Revista Española de Documentación Científica, 21 (4), 402-415.

Delgado, H., y Russell, J. M. (1992). Impact of studies published in the international literature by scientists at the National University of Mexico. Scientometrics, 23 (1), 75-90.

Maz, A.; Torralbo, M.; Vallejo, M., y Fernández-Cano, A. (2007). La producción bibliográfica: un criterio evaluador del rendimiento científico universitario. Revista Tumbaga (2), 93-103.

Moed, H. F. (2005). Citation Analysis in Research Evaluation. Dordrecht (The Netherlands): Springer.

Moya-Anegón, F.; Chinchilla-Rodríguez, Z.; Corera-Álvarez, E.; Gómez-Crisóstomo, M.; González-Molina, A.; Muñoz-Fernández, F. J. (2007). La productividad ISI de las universidades españolas (2000-2004). El profesional de la información, 16 (4), 354-358.

Moya-Anegón, F.; Chinchilla-Rodríguez, Z.; Corera-Álvarez, E.; Vargas-Quesada, F.; MuñozFernández, F., y Herrero-Solana, V. (2005). Análisis de dominio institucional: la producción científica de la Universidad de Granada (SCI 1991-99). Revista Española de Documentación Científica, 28 (2), 170-195.

Torres-Salinas, D.; Delgado López-Cózar, E., y Jiménez-Contreras, E. (2009). Análisis de la producción de la Universidad de Navarra en revistas de Ciencias Sociales y Humanidades empleando rankings de revistas españolas y la Web of Science. Revista Española de Documentación Científica, 32 (1), 22-39.

Van Raan, A. F. J. (2005). Challenges in ranking of universities, First International Conference on word Class Universities. Shanghai: Sahnghai Jia Tong University.

Rev. Esp. Doc. Cient., 33, 4, octubre-diciembre, 582-599, 2010. ISSN: 0210-0614. doi:10.3989/redc.2010.4.757 599 